\title{
Undergraduate laser and optical physics laboratory course at the U.S. Air Force Academy
}

Randall Knize, W. Roc White, Boris Zhdanov

Randall J. Knize, W. Roc White, Boris V. Zhdanov, "Undergraduate laser and optical physics laboratory course at the U.S. Air Force Academy," Proc. SPIE 3831, Sixth International Conference on Education and Training in Optics and Photonics, (16 June 2000); doi: 10.1117/12.388726

Event: Education and Training in Optics and Photonics (ETOP'99), 1999, Cancun, Mexico 


\title{
Undergraduate Laser and Optical Physics Laboratory Course at the United States Air Force Academy
}

\author{
R.J. Knize*, W.R. White and B.V. Zhdanov \\ Laser and Optics Research Center, Department of Physics, \\ US Air Force Academy, Colorado 80840, USA
}

\begin{abstract}
We have developed a new senior-level undergraduate laboratory course at the US Air Force Academy. The students perform six experiments that include; optical modulators, waveguiding, laser kinetics, $\mathrm{CO}_{2}$ lasers, harmonic generation and the measurement of ultrashort laser pulses. These experiments were chosen so that there is an integrated theme of lasers and optics, to teach experimental methods and reinforce fundamental physics concepts.
\end{abstract}

Keywords: Lasers, Optics, Education, Modulators, Optical waveguides, Laser Kinetics, $\mathrm{CO}_{2}$ lasers, Harmonic generation, Optical correlators

\section{INTRODUCTION}

The senior undergraduate laboratory course serves as the capstone experimental course for undergraduate physics students. It is frequently the last structured physics laboratory course that physics majors encounter. In the past, we asked our students to do a number of experiments involving several disciplines, such as plasma, lasers, nuclear, and solid state physics. In the last few years, however, we have restructured this course to create a more integrated curriculum using lasers and optics as the central theme.

We developed this laser laboratory course to accomplish several goals. We selected experiments in lasers and optics due to Air Force and student interests in this field. The presence of lasers and optics in military and civilian applications is widespread. We also wanted this course to reinforce concepts from other physics courses such as quantum mechanics, electricity and magnetism, statistical mechanics and solid state physics. Laboratory experience with laser and optics experiments reinforces ideas learned in these courses and allows for spiral learning of important concepts. We wanted the course to help students develop critical thinking and independent thought. The required work, that is discussed below, helps develop good oral and written presentation skills that are useful latter in life. Overall, we use this course to bridge the gap between textbook learning and independent learning required for research activity.

\section{COURSE OUTLINE}

We selected six experiments in lasers and optics that every student must complete. These experiments are: (1) optical modulators, (2) optical waveguiding, (3) Nd:YAG laser kinetics, (4) $\mathrm{CO}_{2}$ laser characterization, (5) ultrashort laser pulse measurements, and (6) optical harmonics generation ${ }^{1}$. We designed these experiments so that concepts learned in one experiment will be used in subsequent experiments. Typically, we have up to 6 students per class, which we then divide into two groups of two or three students. While the students work in teams, each is independently responsible for the analysis and presentation of the results for each experiment. One group does the experiments in the above numerical order, while the other group does them in the order of 3,4,1,2,6 and 5. In the first two experiments, one group concentrates on optical effects/propagation, while the other group studies laser fundamentals. After the initial two experiments, the groups hear reports on experiments that they will perform in the next round, allowing a greater level of preparation. This approach gives students the opportunity to gain a much greater understanding of the subject material. Following the third and fourth experiments, presentations are once again given, but to a much more informed audience.

Before each lab, we give the students a page of learning objectives, which includes references. Frequently, students will find other texts or research journal articles that they use to understand each lab. During the first lesson of each lab, we give the students an overview of the apparatus and some background on the theory behind each experiment. The students also receive a list of prelab questions that they must answer and turn in by the second lesson of that particular lab. The prelab 
questions get the students thinking about the important concepts for each experiment and also serve as a starting point for preparation of the reports. There is no assigned textbook for this course, so the students must go to the library and perform some reading and research in order to answer these questions. The students are able to complete each experiment in about 10 classroom hours.

\section{EXPERIMENTS}

We will briefly describe the six experiments that the students complete in the semester. Table 1 lists some of key points covered in the experiments. It shows several of the strong ties between experiments, which serves to greatly reinforce learning.

\subsection{Optical Modulators}

In this experiment the students study the principles of modulation of light. Modulators have important technological applications in fiber optics and optical communications. Electro-optical (EOM) and acousto-optical (AOM) modulators are studied. The set-up consists of a He-Ne laser, an $\mathrm{EOM}\left(\mathrm{LiNbO}_{3}\right.$ crystal) with $\mathrm{AC}$ and $\mathrm{DC}$ power supplies, an $\mathrm{AOM}$ (quartz) with a radio frequency generator, a photodetector, an oscilloscope, mirrors, lenses and a polarizer.

In the first part of the experiment the EOM is studied. Applying a voltage to the crystal results in changing of the polarization of light. They determine the half wave voltage for the cell, which is where the electric field polarization is rotated by $\pi / 2$. From this part of the lab, the students develop a good understanding of polarization and how birefringent materials can change the electric field polarization. From the data they calculate the value of the electro-optical coefficient for $\mathrm{LiNbO}_{3}$. They are expected to compare their results with accepted published values.

The AOM is then studied. A sound wave is created in the AOM medium by a piezoelectric transducer resulting in a volume diffraction grating, which diffracts the light beam. The students learn to adjust the position of the rotatable stage supporting the AOM in order to obtain Bragg diffraction. They measure the diffraction angle and its dependence on the acoustic wave frequency. The students learn how sound waves in a material can cause diffraction similar to a grating. Using the experimental data they calculate the speed of sound in quartz.

\subsection{Optical Waveguiding}

This experiment examines the principles of optical waveguiding. Light can be guided in a medium surrounded by materials of lower indexes of refraction. This is the principle used in fiber optics. The apparatus consists of a laser, either a single wavelength Argon ion or HeNe laser, a computer controlled rotation stage, a quartz substrate with a Ge doped silica film of several micron thickness deposited on one side, a photodetector and associated mirrors and lens.

The experiment consists of two parts: the physics of waveguiding, and writing a simple LabView ${ }^{2}$ program to control the experiment. The index of refraction of the thin film is several percent larger than that of the substrate due to the Ge doping. Light will be waveguiding only if the proper boundary conditions are satisfied, which depend on the wavelength, polarization and incident angle. The students first manually rotate the waveguide and substrate using the rotation stage ${ }^{3}$ and observe that at about 6 or 7 angles (for our particular film) the light is waveguided down the thin film. They then spend several lessons developing a LabView program, which will automatically rotate the stage and measure the transmitted light. The program is used to take data for several different wavelengths and for TE and TM light waves. The students learn that boundary conditions are important in the propagation of an electromagnetic wave in a one-dimensional waveguide. They develop an appreciation for the programming necessary to control an experiment. Analysis of the data allows them to determine the index of refraction, its dispersion and the thickness of the doped film.

\subsection{Nd:YAG Laser Radiation Kinetics}

The purpose of this experiment is to acquaint students with solid state lasers and measurement of various laser parameters. The set-up contains a continuously arc lamp pumped Nd:YAG laser ${ }^{4}$ with an acousto-optic Q-switch, a power meter, a frequency doubling crystal $\left(\mathrm{LiNbO}_{3}\right)$, a photodetector, an oscilloscope and electrical spectrum analyzer.

Continuous wave $(\mathrm{CW})$ and pulsed Q-switched regimes of laser operation are investigated in this experiment. First, measurements of the laser power are made as a function of the arc lamp current. For the Q-switched regime, the pulse width 
and the power as a function of the repetition rate are also measured. They use a knife-edge mounted on a translation stage to measure the beam profile both close and far away from the laser. Students can compare these results to data measured by a computer controlled beam profiler, based on a CCD camera. Then they observe relaxation oscillations of laser radiation intensity, measure the frequency of these oscillations and its experimental dependence on pump power. Finally the students observe production of second harmonic light in a $\mathrm{LiNbO}_{3}$ crystal for both the $\mathrm{CW}$ and pulsed regimes.

Students learn about the concept of threshold of the laser operation and the relationship between gain and loss. They are asked to compare the average and peak powers for $\mathrm{CW}$ and pulsed modes. The overall electrical to laser power conversion is calculated. From the decrease in peak pulsed laser power as a function of repetition rate, the energy storage time of $\mathrm{Nd}$ :YAG is also calculated. The beam profile is calculated from the knife edge experiments and the beam divergence is determined. The students are asked to compare their results to a simple model of diffraction. They learn about transverse laser modes. They also observe a large increase in green light produced by second harmonic generation, when Qmodulation is switched on as compared to $\mathrm{CW}$ operation due to the increased peak power.

\section{4. $\mathrm{CO}_{2}$ Laser Characterization}

In this experiment, the students learn how a $\mathrm{CO}_{2}$ laser operates. The apparatus consists of a flowing gas system, $\mathrm{CO}_{2}, \mathrm{~N}_{2}$ and $\mathrm{He}$ gas sources, a power meter and an infrared diffraction grating spectrometer. Infrared sensitive paddles are used to visualize the laser beam and different output couplers are available.

The students are asked to measure the output power as a function of discharge current, various gas mixtures and total pressure. They learn the importance of the three gasses, $\mathrm{CO}_{2}, \mathrm{~N}_{2}$ and $\mathrm{He}$, in optimizing the output power. The laser typically operates in the TEM radial doughnut mode and the students are asked to observe this pattern and identify it. The students are introduced to different transverse laser cavity modes. The output power is measured for a variety of output couplers with transmissions between 50 and $95 \%$. From the measurements with different output couplers, they determine the optimal output mirror and can calculate the gain and loss coefficients and the saturation intensity for the laser. Finally, the output wavelength is measured with the spectrum analyzer. In order to understand how the $\mathrm{CO}_{2}$ laser operates, the students need to learn about quantum mechanics of the different vibrational and rotational modes of the $\mathrm{CO}_{2}$ molecule. The output wavelength can be used to determine which vibrational and rotational lines of the $\mathrm{CO}_{2}$ molecule is lasing.

\subsection{Ultrashort Light Pulses Generation and Measurement Technique}

The continuously pumped solid state Nd:YAG laser with acousto-optic Q-switch and mode locking, and the correlation methodology of ultrashort light pulses duration measurement are studied in this experiment. This experiment builds upon the concepts used in the modulation and Nd:YAG kinetics experiments. The laser used in this experiment produces pulses with width of about 150 psec. The students learn that is difficult to electronically measure these pulse lengths and are introduced to techniques such as using an optical correlator. The set-up contains a Nd:YAG laser ${ }^{5}$ that can be mode-locked and Q-switched, a fast photodiode and an oscilloscope to observe the time dependence of the laser output, an absolute power meter, a frequency doubling crystal and a computer controlled optical correlator for measurement the time duration of the ultrashort light pulses.

In this experiment the students investigate the different possible operation regimes of this laser, such as continuous wave, Q-switched, active mode locking, and simultaneous Q-switched and mode-locking ones. The students measure the average radiation power for the different regimes, the pulse duration and peak power in Q-switched regime and the ultrashort light pulse duration by using correlation methodology. The important part of this experiment is devoted to studying the correlation methodology of measuring the time duration of short light pulses. The correlator is designed similar to a Michelson interferometer in which one of the two beams has a variable time delay with respect to the other beam. The two beams are mixed in the nonlinear KTP crystal and the intensity of the second harmonic obtained in this way is proportional to the product of the instantaneous intensities of two beams. Changing the optical delay between the two beams changes the amount of green light produced. A computer and LabView based software are used for controlling the optical correlator and for storing and analyzing the experimental data. The observed green light power as a function of optical delay distance is described by the correlation function of the intensities of the two beams. The students use the computer to determine which pulse shape function, i.e. Gaussian, Lorenztian, rectangular, etc., best describes the data. They also determine the width of the laser pulses, which are about $150 \mathrm{psec}$, when the laser is modelocked. Using these data and the measured value of laser average power, they calculate the peak power for the ultrashort light pulses. 


\subsection{Optical Harmonics Generation}

Nonlinear optical effects such as second harmonic generation (SHG) and optical mixing are studied in this experiment. Nonlinear optical crystals can be used to sum and difference optical frequencies to create light with different frequencies. The set-up consists of a pulse flash lamp pumped Q-switched Nd:YAG laser ${ }^{6}$, two rotating stages for the nonlinear crystals for second and third harmonics generation respectively, an absolute power meter and three photodiodes for intensity measurements, and a computer for controlling the apparatus and for storing and analyzing the experimental data. Students use three $\mathrm{KDP}$ crystals of $0.5,2$ and $4 \mathrm{~cm}$ lengths and a $2-\mathrm{cm} \mathrm{LiNbO}_{3}$ crystal for studying different features of the second harmonic generation effect. The 4-cm long KDP crystal is used to obtain the third harmonic by sum frequency generation of the fundamental and second harmonic.

The students study the phase matching conditions for SHG, which is where the fundamental and the second harmonic waves travel at the same velocity. They measure the second harmonic power as a function of angle between the beam and the optical axis. Then they study the influence of the crystal type, its length, and input power on the efficiency of SHG. They mix the second harmonic with the fundamental to generate the third harmonic in another KDP crystal that can also be rotated to achieve phase matching. They measure the third harmonic power as a function of fundamental input power. From the data, the students learn that the second harmonic power is dependent on the square of the input power. The angular dependence of the data shows that in order to obtain maximum second harmonic power, it is necessary that the speed of the fundamental and second harmonic be the same. When this occurs, there is constructive addition of the second harmonic waves produced in different parts of the crystal so that phase matching occurs. They also learn that at the phase matching angle, the electric field grows linearly with the crystal length and therefore the power is quadratic with the length. Comparison of results allows determination of the relative second order nonlinear susceptibility of $\mathrm{LiNbO}_{3}$ as compared to KDP. The absolute value of the second harmonic coefficients can also be estimated for these materials. Finally, they determine that third harmonic generation is less efficient and depends on the third power of the input intensity. The students are also introduced to the concept that it is possible to produce laser radiation at certain wavelengths and nonlinear optics can be used to generate different wavelengths.

\section{GRADED WORK}

There are several required reports for each student, in addition to the completed prelabs. Every student is required to present two oral presentations, three written reports, and one poster presentation. These six reports cover each of the six experiments. We try to have each student in the group do a different type of presentation for each experiment, if possible. The course is divided into three blocks, with each block consisting of two experiments. After each block, oral presentations and written reports are collected. Students are able to learn from each other through the oral presentations. After the second written report, we also give the paper to another student for peer review. We grade the student on the quality of the peer review report. The students are then required to incorporate the instructor's and peer's comments into a revised paper. Posters are presented at the end of the semester to the course instructors and to other physics faculty. The requirement for written, oral, and poster presentations develops professional skills that are useful in future academic and professional environments.

\section{LASER SAFETY}

The use of lasers in an undergraduate laboratory always has the associated risk of laser damage to the eye ${ }^{7}$. Therefore, we stress laser safety frequently during the semester. At the beginning of the course, we present a short lecture on laser safety and the use of protective eyewear.

\section{CONCLUSIONS}

The students have generally enjoyed this course. Even though they may have had little prior education in lasers, they have been able to research and learn the basic concepts required for understanding the experiments. We have been able to incorporate concepts from their other theoretical courses into the experiments, and the connection between the various experiments has been extremely beneficial. Students accepted increased responsibility for their learning, and the report, review and listen cycle proved to greatly enhance comprehension. 


\section{ACKNOWLEDGEMENT}

We would like to acknowledge support from the Air Force Office of Scientific Research, the US Air Force Academy and the European Office of Airspace Research and Development (London) for sponsoring the cooperative work with the International Laser Center of Moscow State University.

\section{TABLE 1. KEY POINTS COVERED IN THE EXPERIMENTS}

\section{First and Second Blocks}

1. Optical Modulation

- Wave Propagation

- Polarization

- Wave Plates

- EO/AO Effects

2. Optical Waveguiding

- EM Theory

- TE/TM Modes

- Dispersion

- Argon Laser

- LabView Programming
3. Nd:YAG Laser

- Solid State Laser

- Basic Laser Theory

- Q-switching

- Beam Propagation

4. $\mathrm{CO}_{2}$ Laser

- Gas Laser

- Electrical Pumping

- Output coupling

- Rotational and

Vibrational Transitions

\section{Third Block}
5. Ultrashort Laser Pulses
- QS/ML Output
- SHG
- Autocorrelation
- LabView Application

6. Optical Harmonics Generation

- Q-switched Laser

- SHG and THG (Dependence on angle, Intensity, Crystal type)

- LabView Application 


\section{REFERENCES}

1. Set-ups for the experiments (1), (3), (5) and (6) were developed in cooperation with the International Laser Center of Moscow State University, Moscow, Russia, http://www.ilc.msu.su

2. LabView software, National Instruments Corporation, 11500 N Mopac Expwy, Austin, TX 78759-3504, Telephone: 512794 0100, http://www.natinst.com

3. Programmable Motion Controller PMC200-P, Newport Corporation, 1791 Deere Ave., Irvine, CA 92606 USA, Telephone: 1-800-222-6440, http://www.newport.com

4. Quantronix Series 100 Lasers, Quantronix, 41 Research Way, East Setauket, NY, USA 11733, Telephone: 516273 6900, Fax: 516273 6958, http://www.quantron.com/

5. Quantronix Model 416 Laser. See item 4

6. Quanta-Ray DCR-3, Spectra-Physics, 1335 Terra Bella Avenue, P.O. Box 7013, Mountain View, CA 94039-7013, Telephone: 650961 550, Fax: 650968 5215, http://www.spectra-physics.com

7. H.Weichel, W.A.Danne, L.S.Pedrotti, Laser Safety in the Laboratory, Am. J. Phys., 42, pp. 1006-1013, 1974

* Correspondence: Email: randy.knize@usafa.af.mil; WWW: http://www.usafa.af.mil/dfp/research/lorc/lorchome.htm; Telephone: 719333 4165, Fax: 7193333182 